\title{
- SISTEMA DE INFORMAÇÃO NA GESTÃO DO CUIDADO: ESTUDO DE CASO EM INSTITUIÇÃO DE LONGA PERMANENCIA PARA IDOSOS
}

\author{
Amanda Priscila da Fonseca Baptistini ${ }^{1}$ \\ Celeste José Zanon²
}

resumo

Este estudo trata do sistema de informação (SI) como recurso que visa garantir a disponibilidade e a distribuição de informações imprescindíveis para orientar os profissionais na continuidade dos diferentes cuidados prestados por uma Instituição de Longa Permanência para Idosos (ILPI) do interior de São Paulo. A partir de entrevistas realizadas foi possível analisar as percepções dos profissionais responsáveis pelo cuidado sobre o processo de comunicação vigente. Objetivou-se compreender como se estabelece o auxílio proveniente do SI mantido na instituição quanto a comunicação entre os serviços e

1 Graduada em Gerontologia. Pesquisadora do Grupo de Pesquisa Estratégias de Marketing e Operações em Gerontologia (EMOG) da Universidade Federal de São Carlos (UFSCar). E-mail: baptistiniz@hotmail.com.

2 Graduado em Engenharia Química. Doutor em Engenharia de Produção. Professor Adjunto da Universidade Federal de São Carlos (UFSCar), vinculado ao Departamento de Gerontologia, e coordenador do Grupo de Pesquisa Estratégias de Marketing e Operações em Gerontologia (EMOG). E-mail: celeste@ufscar.br. 
identificar fatores que possam promover uma melhor qualidade do processo comunicacional entre os membros da equipe gestora do cuidado do ponto de vista da disponibilidade, acessibilidade e confiabilidade. Os resultados demonstraram que a ausência de um SI fundamentado em recursos tecnológicos não impediu que a gestão da ILPI sistematizasse as informações inerentes aos cuidados prestados como previsto na legislação. As conclusões indicam que o auxílio do sistema de informação se estabelece por processos de comunicação que perpassam a estrutura hierárquica vertical e horizontalmente. Tais processos procuram gerenciar e suportar as ações individuais e coletivas de trabalho da gestão do cuidado por meio de relatórios, diários de bordo, prontuários e recursos informais como lembretes e cadernetas. Os fatores identificados que podem fomentar melhorias no processo comunicacional são: padronização dos meios de comunicação, rotinização dos registros, avaliação periódica que subsidiem intervenções, conscientização dos riscos de um processo deficiente e a inteligibilidade das informações transmitidas de modo a coibir mal-entendidos que possam afetar a segurança dos residentes.

\section{palavras-chave}

Sistema de Informação. Instituição de Longa Permanência para Idosos. Comunicação. Gestão da Informação. Profissional da Saúde.

O sistema de informação (SI) em saúde é compreendido como recurso para organizar, analisar, bem como para avaliar a eficácia, a eficiência e o alcance dos serviços prestados (WHITE, 1980). Nesse sentido, o sistema de informação como auxílio ao trabalho dos profissionais da saúde, como explica Branco (1996), precisa ser ágil para propiciar estratégias de intervenções individuais e coletivas.

As informações pertinentes à saúde, quando estão disponíveis de imediato, aumentam a confiabilidade do sistema. Perez (2006) ressalta que a informação fidedigna, atual e relevante ao trabalho deve ser disponibilizada a todos os indivíduos e áreas que de fato necessitam ter acesso a ela.

No contexto de uma instituição de longa permanência para idosos (ILPI) é possível mencionar o prontuário como recurso que oferece suporte à equipe 
multiprofissional, visto que a Agência Nacional de Vigilância Sanitária ANVISA (BRASIL, 2004) regulamentou que toda ILPI deve manter um prontuário para cada residente contendo as informações relacionadas ao seu estado de saúde. De acordo com a regulamentação citada, os prontuários necessitam ser preenchidos de forma clara e concisa, devem ser atualizados, assinados e datados pelo responsável em cada atendimento. Adicionalmente, os prontuários precisam estar acessíveis para auditoria por órgãos fiscalizadores e para consultas dos profissionais do cuidado, dos idosos ou de seus responsáveis, desde que asseguradas as condições de sigilo previstos no Código de Ética Médica e o Código de Defesa do Consumidor (BRASIL, 2004).

Petri e Bastos (2006) acreditam que um sistema informatizado de controle de dados do idoso institucionalizado é de suma importância para o armazenamento dos registros das informações de uma ILPI, pois assim é possível explorar todos os benefícios advindos desse controle, exaurindo qualquer possibilidade da perda de informações. Ademais, para os autores supracitados, a informatização dos registros facilita o armazenamento e a recuperação de demasiadas informações.

Dada a importância das informações aos serviços de saúde, Barbosa e Forster (2010) declaram que um sistema de informação de qualidade é aquele em que há um fluxo periódico de registros com preenchimento adequado que ofereça informações completas, bem como relatórios de acompanhamento do cuidado aos profissionais de saúde. Nesse sentido, Bestetti e Chiarelli (2012) entendem que, por meio das informações sistematizadas, é possível organizar, planejar e avaliar uma ILPI para uma melhor compreensão das demandas e dos serviços a fim de promover melhorias do atendimento.

Em síntese, no contexto das ILPIs, o sistema de informação garante a disponibilidade imediata das informações imperativas à atuação dos profissionais da gestão do cuidado. Além disso, direcionam e auxiliam a continuidade dos cuidados prestados, pois colaboram com a qualidade da gestão administrativa, fomentam a colaboração e possibilitam melhorias na comunicação entre os diversos profissionais envolvidos no processo do cuidar.

\section{Objetivo}

O objetivo geral deste estudo é responder a seguinte questão de pesquisa: "como se estabelece o auxílio proveniente do sistema de informação mantido na instituição quanto a comunicação entre os serviços?". O propósito específico é identificar fatores que possam promover uma melhor qualidade de 
comunicação entre os membros da equipe gestora do cuidado, do ponto de vista da disponibilidade, acessibilidade e confiabilidade. Em complementação, foi analisada a trajetória das informações em convergência às necessidades informacionais desses profissionais, haja vista que um sistema de informação inadequado pode possibilitar equívocos quanto a comunicação, oferecendo, dessa forma, riscos à saúde dos idosos.

Risso (2008) esclarece que uma comunicação inadequada entre profissionais devido a falta de ineficiência da informação, aliada a ausência de feedback, pode gerar ansiedade, nervosismo e sentimento de culpa aos envolvidos. Desse modo, a autora alerta que é comum surgirem mal-entendidos e conflitos ocasionados por uma comunicação não compreendida ou reações inesperadas de pacientes, familiares e equipe multidisciplinar. Silva (2006), por sua vez, enfatiza que uma comunicação adequada reduz conflitos, mal-entendidos e possibilita atingir objetivos definidos. Portanto, uma comunicação eficiente depende de informações consistentes, compreensíveis e fidedignas para se evitar erros nos cuidados prestados aos idosos.

\section{Método}

Trata-se de um estudo de caso que procurou desvelar saberes de como e por quê certos fenômenos ocorrem dentro de um contexto específico (NEVES, 1996). A pesquisa realizada buscou compreender como o sistema de informação auxilia a comunicação dos profissionais que atuam na gestão de cuidado de uma ILPI filantrópica, a qual atende aproximadamente trinta idosos, situada no interior Paulista.

Após a aprovação da pesquisa pelo Comitê de Ética em Pesquisa em Seres

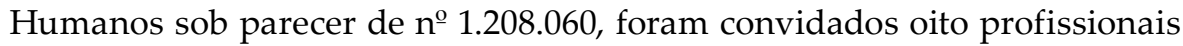
que integram a gestão do cuidado, conforme a Figura 1, para participarem da pesquisa por meio de entrevistas. 
Figura 1 - Organograma da Gestão do Cuidado da ILPI.

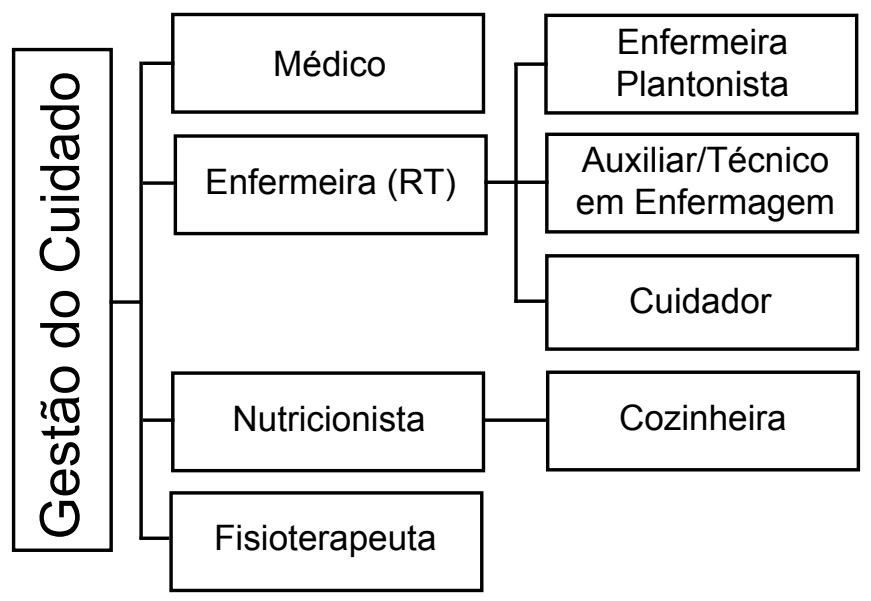

Fonte: Elaborada pelos autores.

As respostas similares e as observações provenientes da coleta dos dados foram analisadas e categorizadas. Determinou-se, por conseguinte, as evidências fundamentais para o desvelamento dos resultados e dos fenômenos relativos ao foco do estudo. Portanto, como preconizam Lima et al. (2012), por meio da estruturação das evidências, constituiu-se cadeias das relações intrínsecas ao objetivo da pesquisa de modo a formar o delineamento das conclusões.

\section{Resultados}

Foram encontradas trinta e nove (39) evidências a partir das percepções dos profissionais do cuidado que propiciaram um melhor entendimento do panorama acerca do sistema de informação como auxílio quanto a comunicação entre os serviços. A partir desse panorama, verificou-se pela evidência E1, um considerável turnover quanto ao quadro de colaboradores que compõe a gestão do cuidado, ou seja, uma significativa rotatividade de profissionais e, por consequência, a evidência E2 mostrou que é recorrente a abertura de vagas para contratações com a intenção de completar o quadro de pessoal. Com exceção da administradora, a rotatividade de funcionários pôde ser identificada pelo fato que, dos profissionais que compõem a gestão do cuidado, aquele com maior tempo de serviço apresenta um período de atuação na ILPI inferior a três anos. 
No que tange a instância da administração, as evidências E3, E4 e E6 esclareceram as formas pelas quais a administradora recebe as informações que orientam sua rotina profissional. A evidência E3 mostrou que a administradora recebe informações sobre os residentes por meio de relatórios elaborados pelos profissionais do cuidado. Do mesmo modo, a evidência E4 revelou o contato direto da mesma com os residentes, apresentando uma importante fonte de informação, enquanto a evidência E6 reforça que a informação chega à administradora por meio de duas principais fontes: pelo profissional e pelo idoso residente. A evidência E5 apresentou que o fluxo de informações entre os serviços se estabelece a partir da interação multiprofissional, principalmente, pela comunicação oral.

A ILPI estudada disponibiliza recursos para registros, consultas e transmissão da informação. No entanto, as evidências E7, E10 e E14 demonstraram que não há suporte digital e tecnológico. Conforme apresenta a evidência E22 na instituição estudada são fornecidos recursos para o registro manuscrito das informações dos cuidados prestados. Constata-se a falta de clareza das informações transmitidas a partir dos registros manuscritos. Letras ilegíveis citadas pelos profissionais mostraram ser um obstáculo para a compreensão das informações.

Os meios de comunicação disponibilizados pela ILPI à gestão do cuidado contemplam, segundo as evidências E11, E12, E17, E20, E25, prontuário multiprofissional, pastas, cadernos de passagem de plantão e ata. Nesse sentido, tais meios que oferecem suporte aos serviços de cuidado foram identificados como fontes de informações formais. Quanto à conceituação, Pletsch (2003, p. 7) esclarece que as informações formais são aquelas que "[...] normalmente possuem a característica de serem bem estruturadas, isto é, de acordo com regras e procedimentos organizacionais". Entretanto, foi verificado, por meio da evidência E21, que não há padronização na denominação dos meios de comunicação utilizados pelos profissionais. Dentre os meios disponibilizados, foi possível observar a partir da evidência E13 que o armazenamento das informações é fragmentado, haja vista que os registros são arquivados em pastas distintas, pertinentes a cada área de cuidado.

Além dos meios de comunicação formais, foram identificados os meios informais, ou seja, fontes que não são organizadas e sistematizadas. Para Pletsch (2003, p. 7), as informações informais "[...] não possuem nenhum caráter oficial". Os profissionais utilizam de meios informais para realizar anotações de cunho pessoal de acordo com a evidência E29. Já a evidência E30 complementa que estes meios favorecem a lembrança de que há registros, os quais não foram efetuados para posterior regularização. 
Em relação às formas de comunicação percebidas pelos profissionais do cuidado, as evidências E8 e E9 apontam que a administradora, ao fornecer informações, faz de modo a associar a oralidade ao registro escrito. No entanto, diante de alguma urgência, não abre mão da flexibilidade que a permite optar pela forma que considerar mais adequada para a situação.

A evidência E16 destacou a passagem de plantão como recurso essencial para orientação dos serviços de cuidados prestados à equipe da enfermagem. Logo, a evidência E31 contribuiu ao detalhar que esta passagem se dá a partir da comunicação verbal, que abarca a oralidade e a escrita. Cabe destacar que as informações escritas são registradas nos cadernos de passagem de plantão já mencionados neste trabalho.

As consultas das informações, conforme expressou a evidência (E18), por vezes, são realizadas com profissionais mais experientes no exercício das funções. Do mesmo modo, a evidência E32 clarifica que não são todos os funcionários que consultam os registros da passagem de plantão a fim de validarem as informações recebidas oralmente.

As evidências E19 e E27 apontaram que a profissional "Responsável Técnica" exerce um papel de articuladora das comunicações durante a distribuição de informações entre os diferentes serviços do cuidado. Ademais, as reuniões são momentos importantes, pois possibilitam a troca de informações conforme detalhou a evidência E26 acerca da comunicação interpessoal.

A evidência E15 mostrou uma baixa interação entre alguns profissionais e a evidência E28 indicou, por meio das percepções, que a maioria dos respondentes não estão satisfeitos com a interação entre áreas. Quanto à acessibilidade das informações, foi possível identificar a partir das evidências E23, E24, E36, E37, E38 que as mesmas ficam disponíveis para consultas a todos profissionais da gestão do cuidado. Tanto os registros dos prontuários quanto os registros da evolução dos residentes são armazenados nas pastas pertinentes a cada área do cuidado.

As evidências elencadas no contexto da ILPI estudada desvelaram fatores que dificultam a comunicação entre os serviços. A evidência E33, por exemplo, traduziu esta problemática, visto que nem sempre há clareza nas informações transmitidas na passagem de plantão, ao passo que as letras ilegíveis apontadas na evidência E34 são as principais responsáveis pela dificuldade mencionada. Nesse mesmo sentido, a dificuldade enfrentada pelos novos profissionais para identificar os idosos também foi mostrada como problema pela evidência E35.

Segundo constatação por meio da evidência E39, as intercorrências restringem o tempo adequado para os registros dos cuidados prestados pelos profissionais da área de enfermagem. Eventos inesperados podem interferir nas 
ações destes profissionais que lidam diretamente com os cuidados de saúde e ser causas da ausência ou da baixa qualidade da informação. Portanto, o tempo parece ser um fator chave, principalmente aos profissionais de enfermagem, pois comumente enfrentam tais intercorrências e, por algumas vezes, podem deixar de registrar informações pertinentes ao cuidado logo após sua realização.

Adicionalmente, embora as informações registradas permaneçam disponíveis para consulta, os profissionais precisam adotar a postura de buscá-las para a validação das mesmas, ou seja, são dependentes da confirmação das informações comunicadas oralmente, de acordo com a evidência E5. Esse fenômeno demostra a importância do profissional de se perceber como parte do sistema de informação que oferece auxílio aos seus serviços.

As análises deste estudo permitiram verificar que uma postura ativa e consciente dos profissionais relacionada ao cuidado não se mostra tão primordial no contexto pesquisado, pois a qualidade dos serviços está fortemente relacionada à comunicação entre os mesmos. Portanto, tal postura deve ser estendida ao processo comunicacional.

\section{Conclusão}

A constituição de cadeias de evidências fundamentadas nas relações entre os resultados, conforme a proposição do método, possibilitou a consecução dos objetivos deste estudo. $\mathrm{O}$ auxílio proveniente do sistema de informação mantido na instituição quanto a comunicação entre os serviços se estabelece por processos de comunicação simples e pouco eficientes que perpassam a estrutura hierárquica vertical e horizontalmente. Tais processos procuram gerenciar e suportar as ações individuais e coletivas de trabalho da gestão do cuidado por meio de relatórios, diários de bordo, prontuários e recursos informais como lembretes e cadernetas. A ausência de um SI fundamentado em recursos tecnológicos não impediu que a gestão da ILPI sistematizasse as informações inerentes aos cuidados prestados como previsto na legislação. Os cinco fatores identificados que podem promover uma melhor qualidade do processo comunicacional entre os membros da equipe gestora do cuidado, do ponto de vista da disponibilidade, acessibilidade e confiabilidade são: a padronização dos meios de comunicação, a rotinização dos registros, a avaliação periódica do sistema de informação que subsidiem intervenções, a conscientização dos riscos de um processo deficiente e a inteligibilidade das informações transmitidas de modo a coibir mal-entendidos que possam afetar a segurança dos residentes. 
O primeiro fator, a padronização dos meios de comunicação, foi definida como um dos fatores por considerar a rotatividade dos funcionários e a escassez de recursos digitais, fatos que impulsionam processos desconexos de comunicação. A ciência da importância de adequações do sistema de informação e das responsabilidades de todos os profissionais que fazem parte dos processos é questão chave para iniciar estudos de padronização. Portanto, trata-se de uma questão de adequação e organização do sistema de informação concomitante a regulares processos de formação pessoal. Uma comunicação adequada entre os serviços pelo auxílio do sistema de informação vigente pode, de fato, promover uma melhor qualidade de vida aos residentes, dentre as condições de fragilidade de cada idoso, considerando que uma efetiva qualidade de cuidado impõe segurança ao atendimento prestado.

A rotinização dos registros - segundo fator - significa compreender que o processo comunicacional está inserido na rotina de cuidar. Portanto, a atividade de registrar uma informação não é menos importante que outra relacionada ao cuidado e consequentemente uma não poderia ser realizada em detrimento da outra. A gestão de serviços deveria garantir a disponibilidade de um período de tempo no qual fosse possível registrar as informações pertinentes ao cuidado logo após sua realização. Assim, o tempo de registro deveria compor o tempo de execução. A sobrecarga de trabalho frequentemente leva o profissional a abrir mão dos registros.

O terceiro fator importante é a avaliação periódica do sistema de informação de modo a subsidiar intervenções. Analisar a adequabilidade dos meios de comunicação e as razões pelas quais os profissionais utilizam meios informais poderia gerar reflexões sobre os elementos constituintes do sistema de informação vigentes na ILPI de modo a minimizar os problemas apontados pela gestão do cuidado. Tais reflexões poderiam suscitar a manutenção de uma comunicação de qualidade entre serviços, afastando o surgimento de erros e falhas que pudessem afetar a segurança dos residentes e prejudicar a qualidade dos atendimentos. Os resultados deste estudo possibilitaram ponderar que se vultuosos investimentos fossem aplicados ao sistema de informação da instituição, pouco avançaria, se o mesmo não fosse alimentado corretamente com dados fidedignos e sem seguir o rigor tecnológico e os critérios necessários que são exigidos. Já o quarto fator - a conscientização dos riscos de um processo deficiente - foi identificado como fator de melhoria devido às seguintes evidências que podem elevar a probabilidade de danos aos residentes: o armazenamento fragmentado de informações, a centralização da intermediação da comunicação e a subestimação dos registros por alguns funcionários. 
O quinto fator, a inteligibilidade das informações transmitidas de modo a coibir mal-entendidos que possam afetar a segurança dos residentes, está diretamente relacionada com a insatisfação cotidiana entre os profissionais das diferentes áreas sobre a interação. Letras ilegíveis e a falta de clareza de informações entre os plantões reforçam a inteligibilidade como um fator que apresenta grande potencial de impactos positivos no processo sem o dispêndio de grandes esforços da gestão da ILPI.

Esse cenário leva a compreensão de que os processos que envolvem os profissionais no que tangem a organização de treinamentos e a padronização dos processos informacionais devem ser reavaliados e aperfeiçoados por profissional capacitado com o intuito de elevar a qualidade e prover segurança aos cuidados oferecidos aos idosos institucionalizados. Assim, tratando-se da gestão comunicacional de uma ILPI, pode-se refletir sobre as competências do profissional gerontólogo acerca da importância dos ajustes, adequações, organização de fatores que abarcam o sistema de informação e o processo de comunicação da gestão do cuidado. Destaca-se que este profissional é capacitado para suprir as lacunas e as carências das demandas que envolvem o processo de envelhecimento nas abrangentes áreas de atuação. Esse contexto pode indicar a atuação de um gerontólogo como um sexto fator de melhorias. Uma vez integrado à equipe multiprofissional e interdisciplinar com intuito de solucionar os problemas apontados, ele poderá acompanhar os profissionais e, entendendo as reais necessidades in loco, intervirá nos processos.

As limitações desta pesquisa fundamentam-se no método e em questões culturais. O trabalho versa sobre um estudo de caso realizado em uma única ILPI, portanto, não se pretende generalizar os resultados bem como as conclusões, visto que a análise foi restrita a um contexto particular. Do ponto de vista cultural é importante lembrar que uma equipe profissional é formada por indivíduos de diversas culturas comunicativas, sejam elas geracionais ou territoriais. Portanto, o que é óbvio para um profissional pode não ser para o outro.

Uma possível contribuição prática deste trabalho reside na conscientização dos gestores de ILPIs sobre a redução dos riscos provenientes de um sistema de informação inadequado e de uma comunicação deficitária por meio do entendimento de como se estabelece o processo comunicacional e da realização de melhorias baseadas nos fatores descritos acima. Tratando-se de contribuições teóricas, os resultados deste estudo podem ser considerados como novos elementos que possibilitem uma melhor gestão do cuidado em ILPIs por meio de melhorias no sistema de informação. Pesquisas futuras podem estabelecer relações entre sistemas informacionais que não envolvam tecnologias e os que são informatizados, analisar a confiabilidade de sistemas 


\title{
em outros modelos organizacionais para idosos e investigar a possibilidade de aplicação de tecnologias móveis na gestão do cuidado em ILPIs.
}

\author{
THE INFORMATION SYSTEM AS SUPPORT \\ TO CARE MANAGEMENT: A CASE STUDY \\ IN A HOME FOR THE AGED
}

\section{abstract}

This present study deals with the information system (IS) as a resource for ensuring availability and the distribution of essential information to orientate and guide practitioners in the continuity of the various care provided by a home for the aged located in the interior of São Paulo State. Based on interviews, it was possible to analyze the perceptions of professionals responsible for care about the current communication process. The objective was to understand how to establish the assistance provided by the IS maintained in the home for the communication between the services and identify factors that can promote a better quality of the communication process between the members of the care management team, considering availability, accessibility and reliability. The results showed that the absence of an IS based on technological resources did not prevent the home management from systematizing the information inherent to the care provided as determined by the legislation. The conclusions indicate that the support of the information system is established by communication processes that cross the hierarchical structure vertically and horizontally. These processes manage and support the individual and collective work actions of care management through reports, logbooks, medical records and informal resources such as reminders and passbooks. The identified factors that can foster improvements in communication are: standardization of communication media, routinization of records, periodic evaluation that subsidize interventions, awareness of the risks of a poor process and the intelligibility of the information transmitted in order to prevent misunderstandings that may affect the safety of residents.

keywords

Information System. Homes for the Aged. Communication. Information Management. Health Personnel. 
BARBOSA, Débora Cristina Modesto; FORSTER, Aldaísa Cassanho. Sistemas de Informação em Saúde: a perspectiva e a avaliação dos profissionais envolvidos na Atenção Primária à Saúde de Ribeirão Preto, São Paulo. Cadernos Saúde Coletiva, Rio de Janeiro, v. 3, n. 18, p. 424-433, mar. 2010.

BESTETTI, Maria Luisa Trindade; CHIARELLI, Tássia Monique. Planejamento criativo em instituições de longa permanência para idosos: estudo de caso em Foz do Iguaçu - PR. Perspectivas em Gestão \& Conhecimento, João Pessoa, v. 2, n. 1, p. 36-51, jan./jun. 2012.

BRANCO, Maria Alice Fernandes. Sistemas de informação em saúde no nível local. Cadernos de Saúde Pública, Rio de Janeiro, v. 2, n. 12, p. 267-270, abr./jun. 1996.

BRASIL. Ministério da Saúde. Agência Nacional de Vigilância Sanitária (ANVISA). Consulta Pública n 41, de 18 de janeiro de 2004. Brasília, DF, 2004. Disponível em: http://www4. anvisa.gov.br/base/visadoc/CP/CP\%5B7626-1-0\%5D.PDF. Acesso em: 1 out. 2015

LIMA, João Paulo Cavalcante et al. Estudos de caso e sua aplicação: proposta de um esquema teórico para pesquisas no campo da contabilidade. Revista de Contabilidade e Organizações, Ribeirão Preto, v. 6, n. 14, p. 127-144, maio 2012.

NEVES, José Luis. Pesquisa qualitativa: características, usos e possibilidades. Caderno de Pesquisas em Administração, São Paulo, v. 1, n. 3, 1996.

PEREZ, Gillberto. Adoção de inovações tecnológicas: um estudo sobre o uso de sistemas de informação na área de saúde. 2006. 227 f. Tese (Doutorado em Administração de Empresas) - Universidade de São Paulo, São Paulo, 2006.

PETRI, Ana Claudia; BASTOS, Laudelino Cordeiro. Sistema Informatizado de Controle de Dados do Idoso Institucionalizado. In: CONGRESSO BRASILEIRO DE INFORMÁTICA EM SAÚDE, 10., 2006, Florianópolis. Anais [...]. Florianópolis: Sociedade Brasileira de Informática em Saúde: UFSC, 2006. p. 401-402.

PLETSCH, Estela. O fluxo de informações como apoio à tomada de decisão: o caso da Central de Atendimento da Telet S.A. 2003. 94 f. Dissertação (Mestrado em Administração) - Universidade Federal do Rio Grande do Sul, Porto Alegre, 2003.

RISSO, Amanda Creste Martins da Costa Ribeiro. A comunicação da suspensão de cirurgias pediátricas: motivos e sentimentos envolvidos no processo. 2008. 89 f. Dissertação (Mestrado em Enfermagem) - Departamento de Enfermagem da Faculdade de Medicina, Universidade Estadual de São Paulo, Botucatu, 2008.

SILVA, Maria Júlia Paes. A comunicação tem remédio: a comunicação nas relações interpessoais em saúde. 4. ed. São Paulo: Loyola, 2006.

WHITE, Kerr. Information for health care: an epidemiological perspective. Inquiry: The Journal of Health Care Organization, Provision, and Financing, v. 17, n. 4, p. 296-312, 1980. 